\title{
Kleinräumige Verteilung von Gesundheitsbedingungen in Städten
}

\author{
Das Beispiel Erlangen
}

\author{
David Spenger ${ }^{1}$ (D) Klaus Geiselhart $^{1}$ (D)
}

Eingegangen: 5. Oktober 2021 / Überarbeitet: 20. Januar 2022 / Angenommen: 21. Januar 2022 / Online publiziert: 7. Februar 2022 (c) Der/die Autor(en) 2022

\section{Zusammenfassung}

Debatten um Umweltgerechtigkeit gehen davon aus, dass ärmere Menschen von höheren gesundheitsrelevanten Umweltbelastungen betroffen sind, als wohlhabende. Untersuchungen in Erlangen zeigen jedoch, dass dies nicht zwangsläufig der Fall ist und nur einzelne sozioökonomisch benachteiligte Gebiete tatsächlich hohen Belastungen ausgeliefert sind. Andere hingegen verfügen durchaus über gute Umweltausstattungen. Zwar lassen sich einzelne besonders privilegierte Gebiete mit geringen Belastungen und guter sozioökonomischer Lage identifizieren aber andere eher wohlhabendere Gegenden zeigen sich als durchaus belastet. Im Zuge neuerer Innenentwicklung gibt es allerdings die Tendenz, dass Umweltressourcen und Umweltbelastungen zunehmend kleinräumiger nach Finanzkraft verteilt werden. Der Artikel entwirft eine leicht übertragbare Methode, die es ermöglicht, anhand der Kernindikatoren Lärmbelastung, Ausstattung mit öffentlichem/privatem Grün und Sozialdaten, Fragen der Umweltgerechtigkeit sehr kleinräumig zu betrachten und im Sinne eines „Frühwarnsystems“ Entwicklungen im Zeitverlauf zu beobachten. Zudem kann der praxisnahe Ansatz als ein Ausgangspunkt für integriertes Verwaltungshandeln gesehen werden, das insbesondere beim Zusammenhang Umwelt und Gesundheit häufig noch defizitär ist.

Schlüsselwörter Umweltgerechtigkeit · Integrierte Stadtentwicklung · Mikrosegregation · Gesundheitsgeographie · Erlangen

\begin{abstract}
Debates on environmental justice assume that poorer people are affected by higher health-related environmental burdens than wealthy people. However, studies in Erlangen show that this is not necessarily the case and that only individual socio-economically disadvantaged areas are actually exposed to high levels of pollution. On the other hand, some have good environmental conditions. Although individual, particularly privileged areas with low burdens and a good socioeconomic situation can be identified, other more affluent areas are also clearly under strain. However, in the course of recent reurbanisation processes, there is a tendency for environmental resources and burdens to be distributed on an increasingly smaller scale according to financial strength. The article presents an easily transferable method that makes it possible to investigate issues of environmental justice on a very small scale and to monitor developments over time in the sense of an "early warning system" by using the core indicators of noise pollution, the provision of public and private urban green, and social data. In addition, the practical approach can be seen as a starting point for integrated administrative action, which is often deficient, particularly in the context of the environment and health.
\end{abstract}

Keywords Environmental justice $\cdot$ Integrated urban development $\cdot$ Microsegregation $\cdot$ Geographies of health $\cdot$ Erlangen

\author{
David Spenger \\ david.spenger@fau.de \\ Klaus Geiselhart \\ klaus.geiselhart@fau.de \\ 1 Institut für Geographie, FAU Erlangen-Nürnberg, \\ Wetterkreuz 15, 91058 Erlangen, Deutschland
}




\section{Einleitung}

Der städtische Raum wird im Zuge von Prozessen wie Kommodifizierung, Flächenversiegelung oder Nachverdichtung immer knapper (Brake 2010). In der Konkurrenz um städtische Ressourcen setzen sich verstärkt finanzstarke Anwohner*innen zu Ungunsten finanzschwächerer durch, was zur Verdrängung von Geringverdienenden aus innerstädtischen Stadtteilen führen kann (u. a. Häußermann 2012). Der Untersuchungsort Erlangen (ca. 114.000 Einwohner*innen, Stand 2020) weist als Wissenschafts- und Wirtschaftsstandort in den letzten Dekaden einen bedeutenden Zuzugsüberschuss von Fachkräften auf, was die Schaffung von Wohnraum und Nachverdichtung zu einem Kernthema der Stadtentwicklung machte. Verschiedene Untersuchungen in Großstädten zeigen, dass sich sozioökonomische Unterschiede dabei nicht nur auf Stadtteilebene, sondern noch kleinräumiger darstellen (Geiselhart et al. 2020). Bei der Stadterneuerung ist soziale Mischung ein erklärtes Ziel und so muss bei größeren Bauprojekten immer ein bestimmter Anteil an Sozialwohnungen geschaffen werden. Diese liegen vorwiegend in Ungunstlagen oder werden gar genutzt, um im Quartier Gunstlagen zu schaffen. Diese kleinräumige Differenzierung geht nicht selten mit einem unterschiedlichen Zugang zu Umweltressourcen bzw. mit ungleichen Umweltbelastungen einher und lässt sich als „umweltbezogene Mikrosegregation“ (Geiselhart et al. 2020) beschreiben.

Ausgehend von Studien aus den Bereichen der Public Health und den sozialwissenschaftlichen Umweltwissenschaften wurden die Zusammenhänge zwischen Umweltfaktoren, Gesundheit und Sozialraum in den vergangenen Jahren auch in Kommunalpolitik und Verwaltung getragen (Bolte et al. 2018). Allerdings sind in Deutschland und auch auf europäischer Ebene nur wenige Städte zu finden, die sich in systematischer und integrierter Art und Weise mit dem Thema Umweltgerechtigkeit beschäftigen. Diesbezüglich stellt die Umweltgerechtigkeitskonzeption des Lan- des Berlin ein richtungweisendes Modellvorhaben dar. Auf der Ebene der 447 Planungsräume (in Berlin „Lebensweltlich orientierte Räume“ genannt) wurde ein zweistufiges Monitoringverfahren angewendet, das mit 5 Kernindikatoren und mehreren Ergänzungsindikatoren zur fachlich-inhaltlichen Untersetzung arbeitete (Klimeczek 2019). Umweltgerechtigkeit lässt sich damit in ressortübergreifende städtische Planungsprozesse integrieren. Diesen Anspruch möchten wir im Folgenden aufnehmen und dabei insbesondere die sozioökonomischen Differenzierungsprozesse operationalisieren, die, wie schon angedeutet, unterhalb der Ebene derartiger Planungsräume ablaufen. Da diese Prozesse vor allem von zunehmender Kommodifizierung in der Wohnungsversorgung ausgehen, werden demografische, ethische und religiöse Segregationsprozesse an dieser Stelle nicht betrachtet.

Ziel dieser Studie war es, mithilfe einer einfachen Methodik die graduelle Verteilung von Umweltfaktoren innerhalb der Stadt Erlangen zu erfassen und Gebiete niedriger, mittlerer und hoher Belastung auszuweisen. Danach können diese auf ihre sozialen Charakteristiken untersucht werden. Dabei wird besonders darauf geachtet, dass die verwendeten Daten für Kommunen leicht zugänglich und abrufbar sind. Mittel- und langfristig erlaubt dies die Einrichtung eines Monitoringverfahrens auf der Mikroebene, welches als „Frühwarnsystem“ umweltbezogener Mikrosegregation entgegenwirken kann.

\section{Methodik: Operationalisierung von Umweltgerechtigkeit}

In einem 3-stufigen Verfahren (siehe Abb. 1) werden zunächst die Kernindikatoren „Lärmbelastung“ und „Stadtgrün“ mithilfe von geografischen Informationssystemen graduell über das Stadtgebiet dargestellt. Diese drücken jeweils einen zentralen gesundheitsfördernden (Environmental Good) und einen gesundheitsschädlichen (Environ-
Abb. 1 Heuristik zur Operationalisierung von Umweltgerechtigkeit

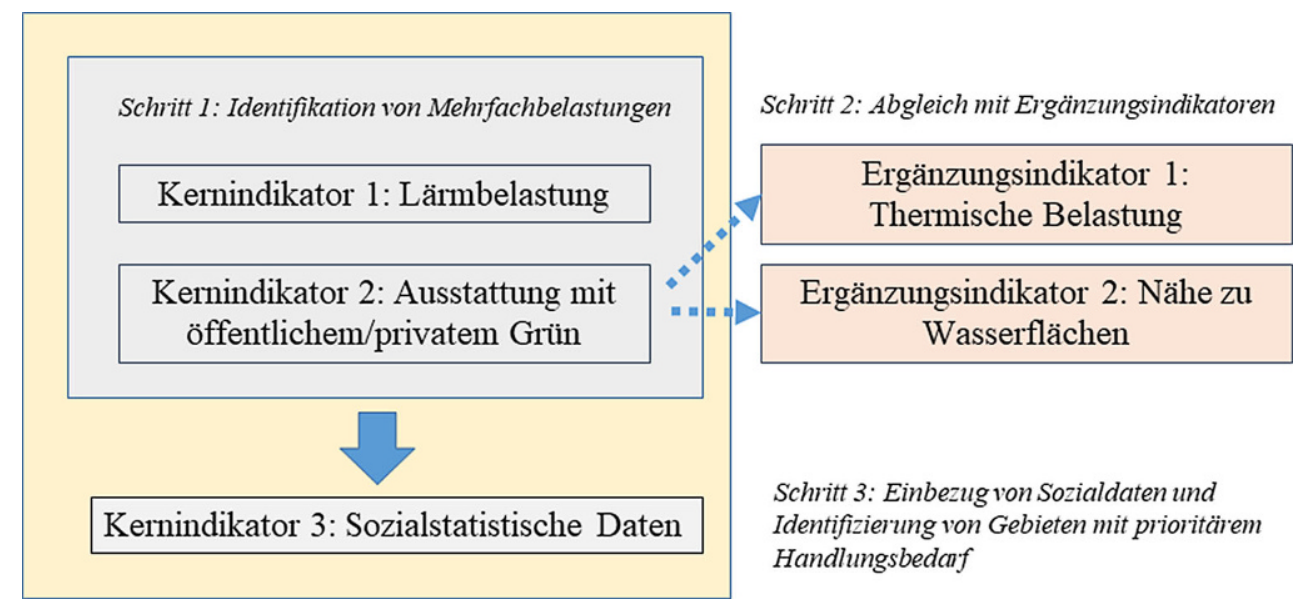


Tab. 1 Ortsspezifische Klassenbildung von Stadtgrün in Erlangen

\begin{tabular}{llll}
\hline Bewertung & Wertebereich & $\begin{array}{l}\text { Anzahl Baublöcke } \\
\text { (bewohnt) }\end{array}$ & $\begin{array}{l}\text { Anzahl Bewohner*innen } \\
\text { (Haupt- und Nebenwohnsitz, } \\
\text { GESAMT: 127.801) }\end{array}$ \\
\hline $\begin{array}{l}\text { Qualität gering/sehr gering } \\
\text { (Fels, überbaute Flächen, vereinzelt Sträucher und/oder }\end{array}$ & $0,0-0,44$ & 438 & 46.341 \\
$\begin{array}{l}\text { Bäume) } \\
\text { Qualität mittel } \\
\text { (Innenhöfe/Grasflächen mit geringerem Baumbestand) }\end{array}$ & $0,45-0,59$ & 524 & 65.764 \\
$\begin{array}{l}\text { Qualität hoch/sehr hoch } \\
\text { (Parks und Gärten mit hohem Baumbestand) }\end{array}$ & $0,60-0,85$ & 210 & 15.696
\end{tabular}

mental Bad) Faktor aus. Die Daten werden auf der kleinsten Gebietsgliederungsebene, die der Häuserblöcke, dargestellt. Zur fachlich-inhaltlichen Untersetzung werden sie in einem zweiten Schritt mittels der Ergänzungsindikatoren ,thermische Belastung“ und „Nähe zu Wasserflächen“ evaluiert. Die Indikatoren wurden wegen ihrer Gesundheitsrelevanz und aus Gründen der Datenverfügbarkeit ausgewählt. Pro Kernindikator werden 3 Klassen gebildet, die im Anschluss die Grundlage für die Berechnung von Einfach- und Mehrfachbelastungen sind.

In einem dritten Schritt werden die Ergebnisse mit einem dritten Kernindikator „sozialstatistische Daten“ verschränkt, um Gebiete mit prioritärem Handlungsbedarf zu identifizieren. Hierbei ist die Auflösung der Sozialdaten maßgeblich. Leider konnte aufgrund datenschutzrechtlicher Bedenken keine Verrechnung auf Häuserblockebene geschehen und es war lediglich eine Diskussion der Ergebnisse aus den ersten beiden Schritten mit spärlich vorhandenen Daten auf Distriktebene möglich. Im Folgenden werden die Kernindikatoren kurz vorgestellt.

\section{Kernindikator 1: Lärm}

Lärm ist nach der Luftverschmutzung der zweitwichtigste Faktor für die umweltbedingte Gesundheitsbelastung in Europa (EEA 2020). Daten über Luftverschmutzung sind nicht flächendeckend verfügbar und die Hauptverursacher von Luftverschmutzung sind in der Regel lärmemittierende Quellen, wie Straßen- und Schienenverkehr. Lärmbelastung ist also ein geeigneter Kernindikator. Da Kommunen in regelmäßigen Zeitabständen Lärmaktionspläne aufstellen müssen, liegt eine gesicherte Datengrundlage vor.

Lärmexposition kann auditive (direkte Schädigungen des Hörorgans) und nicht-auditive (körperliche Stressreaktionen auf Lärm) gesundheitliche Beeinträchtigungen verursachen (vgl. EEA 2020). Letztere können von Schlafstörungen über kardiovaskuläre und metabolische Erkrankungen bis hin zu Beeinträchtigungen der psychosozialen Entwicklung von Kindern oder kognitiven Behinderungen bei Kindern führen (WHO Europe 2018). Zur Bestimmung der Lärmbelastung gibt es verschiedene gesetzliche
Vorgaben und Methoden. Aus Gründen der Vergleichbarkeit orientieren wir uns an der Umgebungslärmrichtlinie der EU. Danach richten sich beispielsweise Ämter auf Europäischer-, Bundes- und Landesebene. Datengrundlage war die Lärmkartierung aus dem Jahr 2017 des Bayerischen Landesamts für Umwelt. Zur Weiterverarbeitung wurde der Index LDEN (Day-Evening-Night) verwendet und die ent-

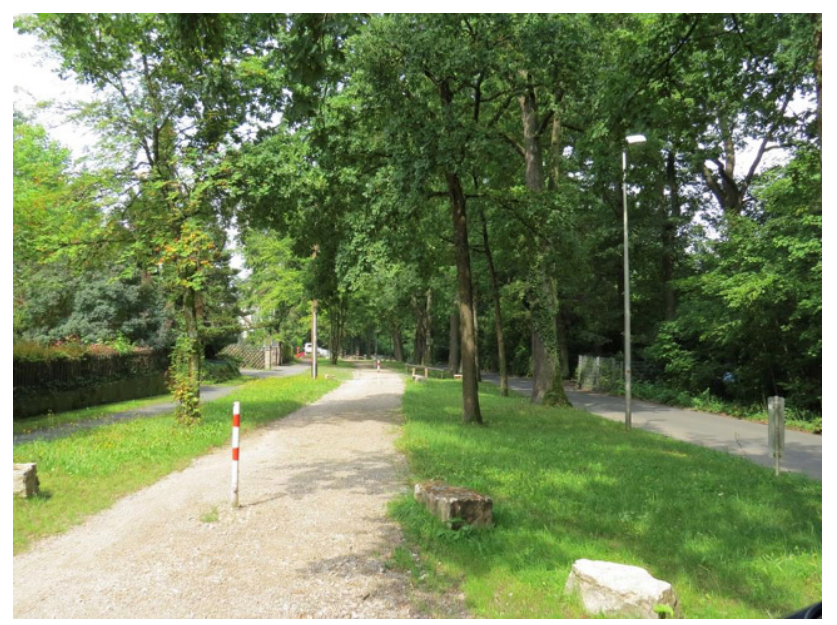

Abb. 2 Wegequalitäten im privilegierten Stadtteil Burgberg

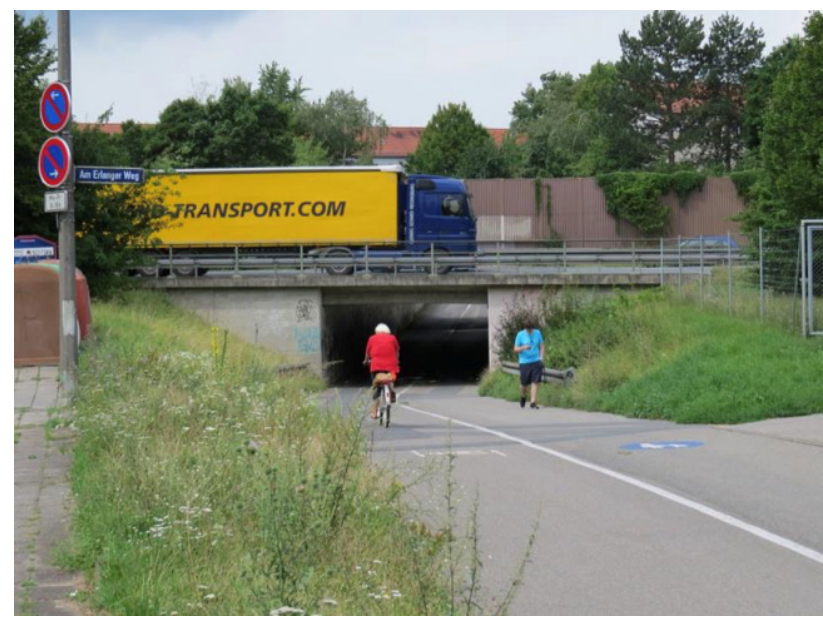

Abb. 3 Wegequalitäten im benachteiligten Stadtteil am Anger 
Abb. 4 Belastungsunterschiede innerhalb der statistischen Distrikte

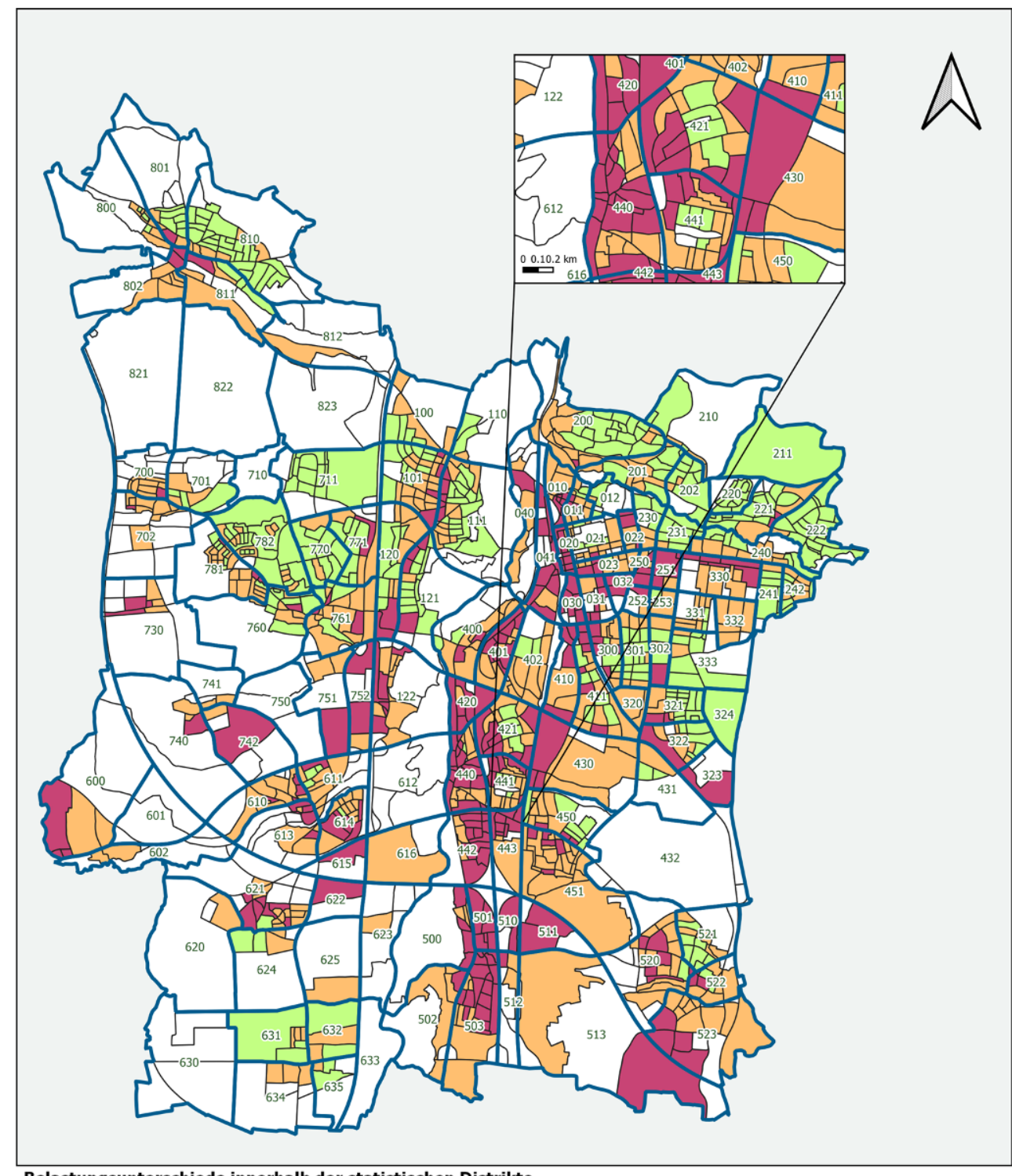

Belastungsunterschiede innerhalb der statistischen Distrikte

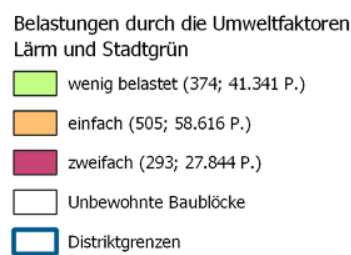

sprechenden Rasterdaten mit den Baublöcken in Erlangen verschnitten.

\section{Kernindikator 2: Ausstattung mit öffentlichem/ privatem Grün}

Während Lärm als Umweltstressor die menschliche Gesundheit beeinträchtigt, sind Stadtgrün und Stadtblau gesundheitsförderliche Ressourcen. Stadtgrün hat positive gesundheitliche Wirkungen sowohl auf psychisch-mentaler

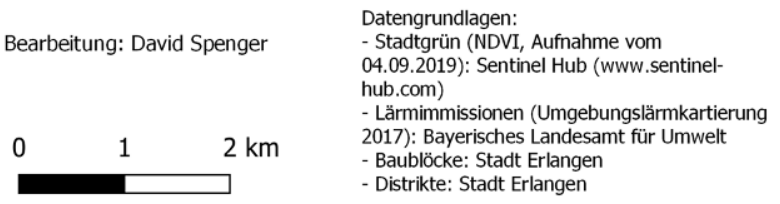

(z.B. Bringslimark et al. 2007) als auch auf physisch-körperlicher Ebene (z.B. WHO 2016). Dies ist insbesondere für Einkommensschwächere und Benachteiligte von großer Bedeutung (Ward Thompson et al. 2016). Stadtgrün und Stadtblau können auch Umweltstressoren abschwächen, etwa bezüglich Straßenverkehrslärm (de Coensel et al. 2011) oder bei klimatischen Extremen (Völker et al. 2013). Bedeutsam sind besonders dezentrale Begrünungsmaßnahmen in unmittelbarer Wohnortnähe. Angenommen wurde daher eine hohe Korrelation von Flächenversiegelung und damit 
Tab. 2 Strukturierung der bewohnten statistischen Distrikte in Erlangen nach Umweltbelastungen

\begin{tabular}{|c|c|c|c|c|c|c|}
\hline & & \multicolumn{3}{|c|}{ Interne Heterogenität $^{b}$} & \multirow[t]{2}{*}{ Summe } & \multirow[t]{2}{*}{ Anteil (\%) } \\
\hline & & Gering ausgeprägt & Mittel ausgeprägt & Stark ausgeprägt & & \\
\hline \multirow{3}{*}{$\begin{array}{l}\text { Grad der } \\
\text { Belastung }\end{array}$} & Wenig belastet & 14 & 8 & 7 & 29 & 25,2 \\
\hline & Einfach belastet & 18 & 16 & 17 & 51 & 44,4 \\
\hline & Zweifach belastet & 17 & 14 & 4 & 35 & 30,4 \\
\hline Summe & - & 49 & 38 & 28 & 115 & - \\
\hline Anteil (\%) & - & 42,6 & 33,0 & 24,4 & - & 100 \\
\hline
\end{tabular}

${ }^{a}$ Eine Zuordnung wird dann vorgenommen, wenn die meisten Baublöcke innerhalb des Distrikts der jeweiligen Belastungsstufe entsprechen. Die Zuordnung spiegelt demnach eine Tendenz für eine Belastungsstufe wider

${ }^{b}$ Eine geringe Ausprägung liegt vor, wenn innerhalb eines Distrikts nur vereinzelte Baublöcke eine andere als die dominante Belastungsstufe haben. Eine mittlere Ausprägung besteht dann, wenn viele Baublöcke eine andere Belastungsstufe aufweisen. Bei einer stark ausgeprägten Heterogenität sind alle Belastungsstufen vorhanden

verbundener Hitzebelastung, was sich durch die Hinzunahme des Ergänzungsindikators „thermische Belastung“ empirisch auch zeigte. Um zusätzlich die Wichtigkeit von Stadtblau zu berücksichtigen, wurden die Ergebnisse mit dem Ergänzungsindikator „Nähe zu Wasserflächen“ validiert.

Fernerkundung birgt ein großes Potenzial für die Umweltgerechtigkeitsforschung (Weigand et al. 2019). Zur Darstellung von Qualität und Verteilung von Stadtgrün innerhalb des Stadtgebietes wurde der Normalized Difference Vegetation Index (NDVI) verwendet und ein Szenario aus der vegetationsreichen Jahreszeit gewählt (04.09.2019). Um den lokalen Bedingungen gerecht $\mathrm{zu}$ werden, wurde keine universelle, sondern eine ortsspezifische Klassifizierung vorgenommen (siehe Tab. 1). Die Klassengrenzen richteten sich nach bestimmten Ausstattungen mit Stadtgrün im Stadtgebiet und teilten die verschiedenen Freiflächen in 3 Kategorien ein: Hohe NDVI-Werte lassen sich in Parks und an Wegen mit hohem Baumbestand finden (siehe Abb. 2), charakteristisch für mittlere Werte sind Flächen mit geringerem Baumbestand, wie beispielsweise Innenhöfe, und auf Flächen mit geringen NDVI-Werten lassen sich eher vereinzelte Sträucher und/oder Bäume erkennen (siehe Abb. 3).

\section{Zwischenergebnisse: Heterogene Gesundheitsbedingungen innerhalb des Stadtgebiets}

Die beiden Kernindikatoren zeigen auf Baublockebene, dass die Gesundheitsbedingungen in Erlangen kleinräumlich sehr variieren (siehe Abb. 4). Innerhalb einiger Distrikte kommen alle Belastungsstufen vor, während manche Distrikte durchaus intern homogen sind. Es bietet sich daher an, die 115 bewohnten Distrikte hinsichtlich ihrer Belastungsunterschiede und internen Heterogenität zu differenzieren (siehe Tab. 2). So können von den 115 bewohnten Distrikten im Stadtgebiet 29 als wenig belastet charakterisiert werden. Darunter fallen 14 Distrikte, die eher homogen belastet sind, 8 weisen eine mittlere Heterogenität auf und 7 sind sehr heterogen.

Von den 115 bewohnten Distrikten können 35 als zweifach belastet angesehen werden. Davon weisen 17 eine geringe, 14 eine mittlere und 4 eine stark ausgeprägte interne Heterogenität auf. Diese 17 Distrikte mit einer nahezu flächendeckenden zweifachen Belastung haben entsprechend schlechte Gesundheitsbedingungen und können nach Umweltaspekten als Gebiete mit prioritärem Handlungsbedarf identifiziert werden (z. B. 030, 041, 250, 251, 420, 440, 442, $501,503)$.

\section{Soziale Lage und Umweltgerechtigkeit}

Die bisherigen Ausführungen bezogen sich rein auf die Umweltbelastungen. Um Aussagen zu Umweltgerechtigkeit treffen zu können, müssen die Ergebnisse nun mit den zur Verfügung stehenden Sozialdaten verschränkt werden (siehe Abb. 5).

\section{Soziale Lage als Indikator}

Städte sind sozioökonomisch segregiert und Armut macht nachweislich krank (Tabert 1999). Letzteres ist keine Frage des individuellen Lebensstils. Das Fehlen finanzieller Mittel erlaubt nicht nur weniger Selbstfürsorge, sondern schwächt auch gesunderhaltende Widerstandsressourcen (Antonovsky 1996). Die sozialen Verhältnisse sind im Sinne des biopsychosozialen Krankheitsmodells (Egger 2005) als ein erhöhtes Morbiditäts- und Mortalitätsrisiko zu betrachten. Soziale Benachteiligung wird demnach als dritter Belastungsfaktor gewertet.

Aus Gründen der Datenverfügbarkeit waren leider keine Analysen auf Baublockebene möglich. Dies hätte es erlaubt, kleinräumige Belastungsareale auszuweisen. An dieser Stelle möchten wir bemerken, dass sich die datenschutzrechtlichen Bedenken durchaus hätten ausräumen lassen, 
Abb. 5 Anzahl SGBII-Empfänger*innen auf Distriktebene

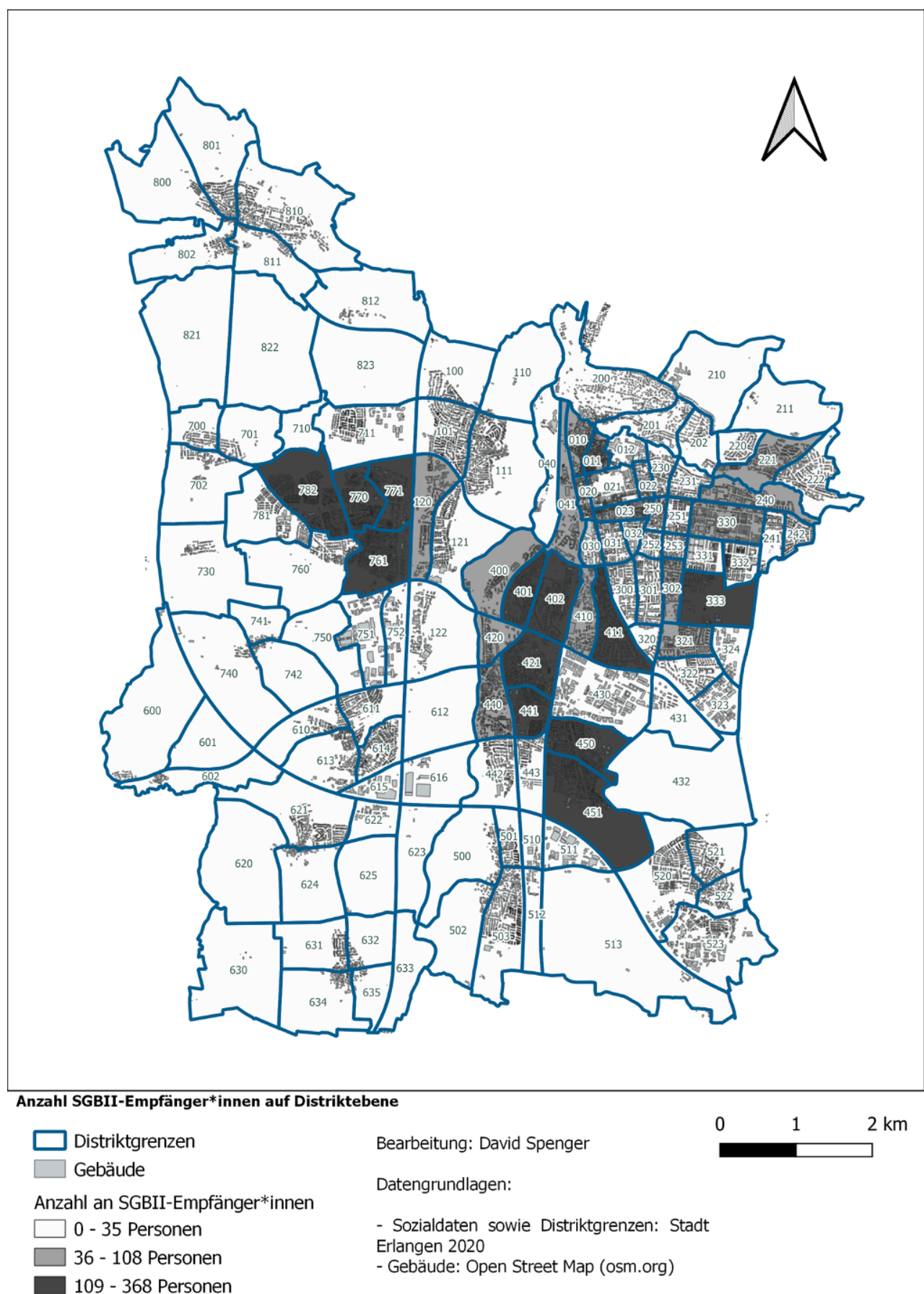

denn aufgrund der aggregierten Daten wären am Ende keine individuellen Bewohner*innen identifizierbar geworden. In diesem Zusammenhang sind in Zukunft gezielte Absprachen innerhalb der Stadtverwaltung und die Entwicklung geeigneter Datenschutzvereinbarungen essenziell. Alternativ lassen die vorhandenen Sozialdaten aber zumindest eine Diskussion von Umweltgerechtigkeit auf Distriktebene zu. Dabei sind insbesondere die Empfänger*innen von Sozialhilfe nach SGBII interessant, weil sie auch vorwiegend diejenigen sind, denen die durch Mikrosegregation geschaf- fenen Wohnungen in Ungunstlagen als geförderte Wohnungen zugeteilt werden.

\section{Ergebnisse Umweltgerechtigkeit}

Es fällt auf, dass die zuvor beschriebenen Distrikte mit prioritärem Handlungsbedarf sich nur bedingt mit der Verteilung der SGBII-Empfänger*innen decken. So sind in einigen eher umweltbelasteten Distrikten (z.B. 030, 031, $250,251,501,503$ ) eher wenig Sozialhilfeempfänger*innen 
wohnhaft. Entsprechend ist auf der Basis der Datenlage keine zusätzliche Konzentration sozioökonomisch benachteiligter Menschen zu vermuten. Anders stellt sich die Situation in den Distrikten im Zentrum und südlichen Zentrum dar (v.a. 401, 402, 421, 441). Dort ist teilweise sowohl eine hohe Umweltbelastungsstufe als auch eine überdurchschnittlich hohe Anzahl an SGBII-Empfänger*innen vorhanden. Bereiche im westlichen Stadtteil Büchenbach (761, 770, 771), die häufig als ,soziale Brennpunkte“ beschrieben werden (Nordbayerische Nachrichten 2019), sind hingegen nur von geringer Umweltbelastung betroffen. Kleinräumige Umweltungerechtigkeiten lassen sich dort nicht oder nur sehr vereinzelt vermuten $(770,771)$. Es gibt Bereiche im Stadtgebiet, in denen wir weder soziale Problemlagen noch ungünstige Umweltbedingungen aufzeigen können (z.B. 200, 222, 711).

\section{Diskussion der Ergebnisse}

Sozioökonomisch schwächere Bevölkerungsgruppen leben in Erlangen häufig in alten Werkssiedlungen oder Trabantenstädten der Moderne. Gegenüber heutigen Planungen verfügen diese Siedlungen noch über verhältnismäßig weite Freiflächen, auf denen sich in einigen Fällen über die Jahre ein wertvoller Baumbestand entwickelt hat. Damit bieten sie verhältnismäßig grüne und gesunde Lebensverhältnisse. Sicherlich gibt es auch in diesen Gebieten benachteiligte Wohnlagen, etwa an Straßen. Solange die Wohnraumbewirtschaftung noch egalitären Ansprüchen genügt, können sich langjährige Bewohner*innen auf bessere Lagen bewerben. Wenn heute aber im Zuge des Stadtumbaus soziale Mischung angestrebt wird, dann bekommen Investor*innen die Auflage, einen bestimmten Anteil an Sozialwohnungen zu schaffen. Im Sinne der Profitmaximierung werden diese Wohnungen nicht selten in Baukörpern untergebracht, die der Abschirmung anderer, begünstigter Lagen gegenüber Umweltbelastungen dienen (Geiselhart et al. 2020). In begünstigten Lagen lassen sich dann auch hochpreisige Wohnungen verwirklichen. Der Zugang ärmerer Menschen zu weniger belasteten Lagen wird dadurch stark eingeschränkt. Um derartige Tendenzen darstellen zu können, wäre eine deutlich kleinräumigere Verfügbarkeit der Sozialdaten notwendig.

\section{Fazit}

Die entwickelte Methode zur Darstellung von Umweltgerechtigkeit ist technisch relativ einfach und auch auf andere Kontexte übertragbar. Die Auflösung, in der sozialstatistische Daten für das Verfahren verfügbar gemacht werden, bestimmt dabei die Genauigkeit, mit welcher Strukturen der Umweltungerechtigkeit identifiziert werden können.
Aus Gründen der Datenbeschaffung und des Datenschutzes weist diese Untersuchung Lücken auf, die durch geeignete Datenschutzvereinbarungen ausgeräumt werden können. Dennoch erlaubt sie die rasche Erstellung eines räumlich differenzierten Lageberichts zur Verteilung von Umweltbedingungen. Zur Beurteilung der Entwicklung erscheint es darüber hinaus sinnvoll, Zeitreihen zu erstellen, wie auch in Einzelfällen die Aussagen mittels qualitativer Methoden vor Ort zu kontrollieren.

Danksagung Unser Dank gilt allen Mitarbeiter*innen der Stadtverwaltung Erlangen, die stets konstruktive Hinweise lieferten und ihren Erfahrungsschatz in der praktischen Tätigkeit mit uns teilten. Außerdem sind wir für die Bereitstellung von Datengrundlangen dankbar. Danken möchten wir auch dem Bayerischen Landesamt für Umwelt, das uns die Datengrundlage für die Berechnung der Lärmbelastung zur Verfügung stellte.

Funding Open Access funding enabled and organized by Projekt DEAL.

Open Access Dieser Artikel wird unter der Creative Commons Namensnennung 4.0 International Lizenz veröffentlicht, welche die Nutzung, Vervielfältigung, Bearbeitung, Verbreitung und Wiedergabe in jeglichem Medium und Format erlaubt, sofern Sie den/die ursprünglichen Autor(en) und die Quelle ordnungsgemäß nennen, einen Link zur Creative Commons Lizenz beifügen und angeben, ob Änderungen vorgenommen wurden.

Die in diesem Artikel enthaltenen Bilder und sonstiges Drittmaterial unterliegen ebenfalls der genannten Creative Commons Lizenz, sofern sich aus der Abbildungslegende nichts anderes ergibt. Sofern das betreffende Material nicht unter der genannten Creative Commons Lizenz steht und die betreffende Handlung nicht nach gesetzlichen Vorschriften erlaubt ist, ist für die oben aufgeführten Weiterverwendungen des Materials die Einwilligung des jeweiligen Rechteinhabers einzuholen.

Weitere Details zur Lizenz entnehmen Sie bitte der Lizenzinformation auf http://creativecommons.org/licenses/by/4.0/deed.de.

\section{Literatur}

Antonovsky A (1996) The salutogenic model as a theory to guide health promotion. Health Promot Int 11(1):11-18

Bolte G, Bunge C, Hornberg C, Köckler H (2018) Umweltgerechtigkeit als Ansatz zur Verringerung sozialer Ungleichheiten bei Umwelt und Gesundheit. Bundesgesundheitsblatt Gesundheitsforschung Gesundheitsschutz 61:674-683. https://doi.org/10. 1007/s00103-018-2739-6

Brake K (2010) „Reurbanisierung“ - janusköpfiger Paradigmenwechsel, wissensintensive Ökonomie und neuartige Inwertsetzung städtischer Strukturen. In: Belina B, Gestring N, Müller W, Sträter D (Hrsg) Urbane Differenzen - Disparitäten innerhalb und zwischen Städten. Westfälisches Dampfboot, Münster, S 69-96

Bringslimark T, Hartig T, Patil G-G (2007) Psychological benefits of indoor plants in workplaces: Putting experimental results into context. HortScience 42:581-587

de Coensel B, Vanwetswinkel S, Botteldooren D (2011) Effects of natural sounds on the perception of road traffic noise. J Acoust Soc Am 129(4):148-153

EEA (2020) Healthy environment, healthy lives: How the environment influences health and well-being in Europe. EEA Report, Bd. 21/2019. EEA, Kopenhagen 
Egger JW (2005) Das biopsychosoziale Krankheitsmodell. Grundzüge eines wissenschaftlich begründeten ganzheitlichen Verständnisses von Krankheit. Psychol Med 16(2):3-12

Geiselhart K, Eisemann C, Feick F, Kammerbauer S (2020) Poor Doors in Erlangen. Umweltbezogene Mikrosegregation unter Bedingungen der Reurbanisierung. sublurban 8(1/2):77-98

Häußermann H (2012) Wohnen und Quartier: Ursachen sozialräumlicher Segregation. In: Huster E-U, Boeckh J, Mogge-Grotjahn H (Hrsg) Handbuch Armut und Soziale Ausgrenzung. Springer, Wiesbaden, S 336-349

Klimeczek H (2019) Umweltgerechtigkeit im Land Berlin. Entwicklung einer ressortübergreifenden und quartiersbezogenen Strategie in der Umsetzung. Land Berlin

Nordbayerische Nachrichten (2019) Nachverdichtung im Erlanger „Brennpunkt“ Odenwaldallee (23.10.2019). https://www. nordbayern.de/region/erlangen/nachverdichtung-im-erlangerbrennpunkt-odenwaldallee-1.9450349. Zugegriffen: 24. Sept. 2021

Tabert G (1999) Armut und Gesundheit. Soziale Dimension von Krankheit vernachlässigt. Dtsch Arztebl 96(12):756-760
Völker S, Baumeister H, Claßen T, Hornberg C, Kistemann T (2013) Evidence of the temperature-mitigating capacity of urban blue space-A health geographic perspective. Erdkunde 67(4):355-371

Ward Thompson C, Aspinall P, Roe J, Robertson L, Miller D (2016) Mitigating stress and supporting health in deprived urban communities: the importance of green space and the social environment. Int J Environ Res Public Health 13(4):440. https://doi.org/ 10.3390/ijerph13040440

Weigand M, Wurm M, Dech S, Taubenböck H (2019) Remote sensing in environmental justice research-A review. Int J Geoinf. https:// doi.org/10.3390/ijgi8010020

WHO (2016) Europe urban green space interventions and health: A review of evidence. World Health Organization Regional Office for Europe, Copenhagen

WHO Europe (2018) Environmental noise guidelines for the European region. World Health Organization Regional Office for Europe, Copenhagen. http://www.euro.who.int/_data/assets/pdf_ file/0008/383921/noise-guidelines-eng.pdf?ua=1. Zugegriffen: 8 . Apr. 2021 